\title{
PC/PMMA 共混物界面相行为的研究
}

\author{
寇喜春 姜振华 马荣堂 张丽梅 \\ (吉林大学化学系,长春 130023) \\ 郑荣光董风霞 \\ （吉林大学分析测试中心，长春 130023)
}

\section{关词共混物、界面相、自由体积、作用力}

聚甲基丙烯酸甲酯 (PMMA) 是脆性高聚物之一, 在拉伸过程中呈现典型的脆性断裂, 但 是当 PMMA 和聚碳酸酯 (PC) 以一定比例熔融或溶液共混时, 试样的力学性能都在两纯组 分性能的加合值之上. 这一令人感兴趣的结果由 Koo 等人发现 ${ }^{[1]}$. 类似的聚合物对, 还有 聚碳酸酯/丙烯腈一苯乙烯共聚物 (PC/AS), 聚酚氧/聚甲基丙烯酸甲酯 (Phenoxy/PMMA), 聚酚氧/聚苯乙烯 (Phenoxy/PS) ${ }^{1)}$.Koo 等认为, 当满足以下三条件时, 共混物有增㓞效应, 即 （1）两组分扬氏模量、泊松比不同；（2）共混物的压缩应力高于脆性高聚物的压缩应力值； (3) 脆性分散相的粒径在 $1-2 \mu \mathrm{m}$ 之间.

Kalauchi 认为, 当以脆性高聚物为分散相, 韧性高聚物为连续相的共混物在拉伸时, 在界 面相将产生一个静压强, 当这一压强值超过分散相的脆性一㓞性转变临界压强时, 脆性分散相 可以出现㓞性形变 ${ }^{[3]}$.

对于脆性高聚物作为分散相的增韧机理, 进一步解释并不多见. 大家知道, 当共混物为 部分相容时, 分子链段间有较强的作用力, 共混物的密度增加, 力学性能也有不同程度提高, 但 若直观测定分子链间的作用力; 入小是困难的. 我们采用自旋探针将其均匀混人共混物中, 并 通过在不同组分共混物中的自旋探针的 ESR 谱, 比较了各向同性与各向异性成分的比值变 化, 表征共混物的两种分子链段间作用力大小, 界面相自由体积的变化, 从而可对共混物的界 面相行为进行微观研究.

\section{1 实 验}

\section{1 原材料}

PC: 常州树脂厂生产, Mn 为 35,000; PMMA: 哈尔滨有机玻璃厂生产, $\mathrm{Mn}$ 为 14,000; 自旋探针 TEMPOL $(2,2,6,6$-四甲基哌啶)试剂级.

\section{2 样品制备}

将 PC, PMMA 粒料按 PC/PMMA 组分比为 $90 / 10$ 和 70/30 的配比用二氯甲烷溶解, 并制成固含量 $15 \%$ 的稀溶液, 用同样方法分别制成 PC 和 PMMA 纯组分的溶液, 同时把 $1 \%$

1992-11-24 收稿.

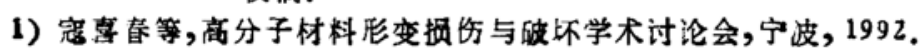


的 TEMPOL 二氯甲烷溶液均匀的混人上面四种样品溶液中, 制成厚度为 $1-2 \mathrm{~mm}$ 的薄膜, 真空干燥后用去离子水洗涤、干燥。

\section{3 测试}

ESR 测量是在 BRUKER ER200D ESR 谱仪上进行, $\mathrm{X}$ 波段, 调制频率 $100 \mathrm{kHz}$, 变温 装置用谱仪的 ER4111 VT 单元.

共混物动态力学性能在吉林大学计算机系研制的 GDP 扭辫仪上进行, 拉伸性能在长春 材料试验机厂产 BL1000 电子拉力机上进行.

\section{2 结果与讨论}

\subsection{ESR 橧分析}

自旋探针 TEMPOL 在不同样品中受各自材料与组份比的分子链段运动及周围环境的影 响, 使其 ESR 谱的形状,各种参数随之发生变化, 这是因为氮-氧自由基的 $\boldsymbol{g}$ 张量和 $\mathbf{A}$ 张量是 由各向同性和各向异性两部分组成，在低粘度稀溶液中只表现出各向同性部分，但随着体系 粘度及分子链刚性程度增加, 分子运动减慢, 各向异性成分遂渐增加 ${ }^{[4]}$. 图 1 的谱图 是 $\mathrm{PC}$, PMMA，PC/PMMA (90/10,70/30) 四种样品在 $350 \mathrm{~K}$ 温度下测定的.

从图中看到在同一温度条件下, PC, PMMA, PC/PMMA (90/10), PC/PMMA (70/ 30) 四种 ESR 谱图两边线之间的距离 $2 A_{x x}$ 不相同,其中 PC/PMMA(90/10 及 70/30) 的距 离略大于 PC 及 PMMA, 说明这两种共混物内的自由体积小于 PC 及 PMMA; 这种现象 可以用 PC 和 PMMA 分子链段间有较强的作用力来解释.

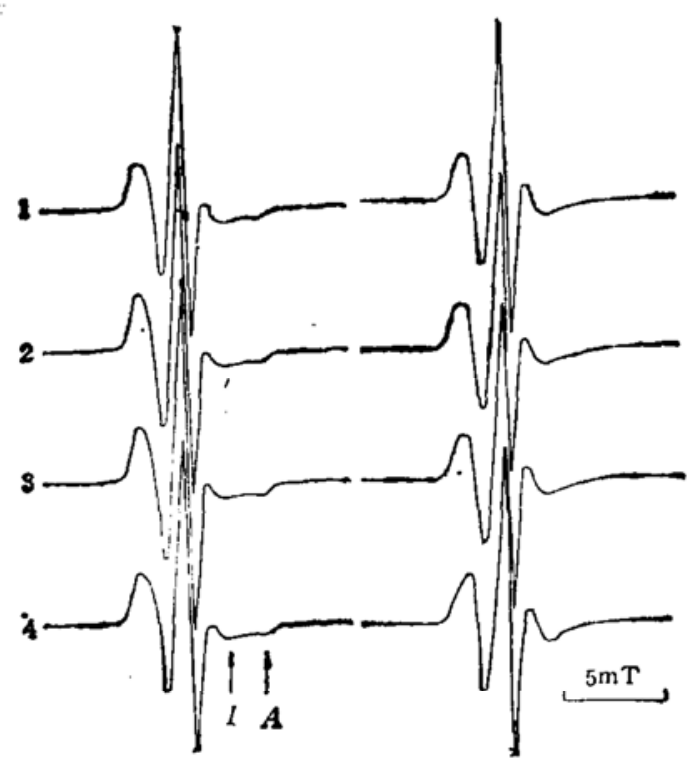

图 1 㷋氧自由基在共混物中的 ESR 谱 $(350 \mathrm{~K})$

1 为 $\mathrm{PC}, 2$ 为 $\mathrm{PC} / \mathrm{PMMA}(90 / 10)$, 3 为 $\mathrm{PC} / \mathrm{PMMA}(70 / 30), 4$ 为 PMMA

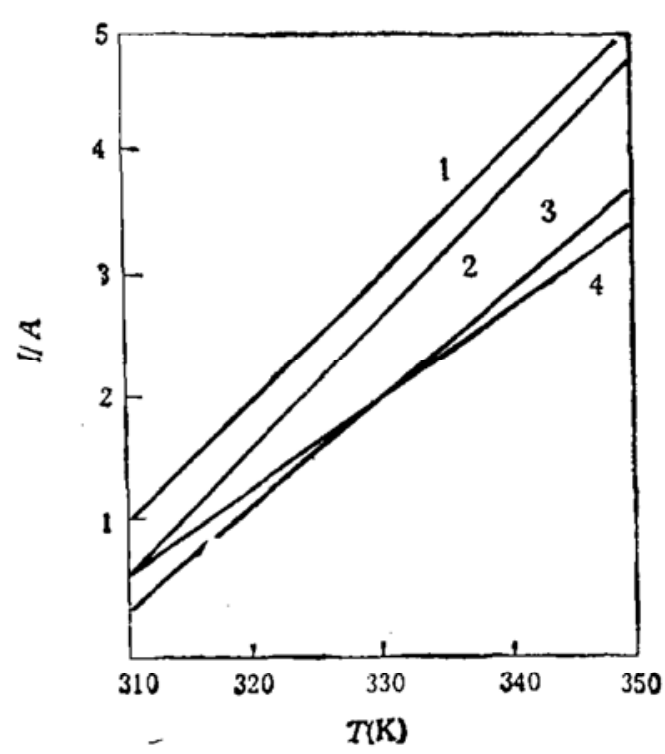

图 2 共混物的 $\boldsymbol{I} / \boldsymbol{A}-\boldsymbol{T}$ 关系曲线 1 为 PC/PMMA $(100 / 0), 2$ 为 PC/PMMA $(0 / 100)$, 3 为 PC/PMMA $(90 / 10), 4$ 为 PC/PMMA $(70 / 30)$

对于自旋探针分子的 ESR 谱来说, 高场线对分子中 $\mathrm{N}-\mathrm{O}$ 键的运动状况十分敏感, 在 一定的温度范围内, 谱中代表各向异性成分的峰与代表各向同性成分的峰没有重叠,所以可以 用它们的峰高表示谱中的不同成分,在图 1 中用 $I$ 表示谱的各向同性成分, 用 $A$ 表示谱的各向 
异性成分,并比较了 $310 \mathrm{~K}-350 \mathrm{~K}$ 之间的 $I / A$ 值, 列入表 1 中, $I / A$ 对温度作图绘于图 2. 从图中看到四种样品 $l / A$ 值都随温度升高而增加, 表明温度升高, 探针分子所处环境的自由 体积增大, 链段刚性减弱. 在同一温度下, 比较 PC, PMMA, PC/ PMMA ( $90 / 10$ 及 70/30) 四种样品的 $1 / A$ 值, 可以看到两个共混物样品 $1 / A$ 值都略低于 PC 和 PMMA. 这表明在 共混物界面相由于不同分子链段之间作用力加强, 使链段刚性增大, 自由体积减小. 可以想 像, 这一作用力的增强会使共混物密度增大, 其力学性能相应提高.

表 1 共混物样品在不同温度下的 $I / A$ 比值

\begin{tabular}{l|c|c|c|c}
\hline I/A 比值 & PC & $\begin{array}{c}\text { PC/PMMA } \\
(90 / 10)\end{array}$ & $\begin{array}{c}\text { PC/PMMA } \\
(70 / 30)\end{array}$ & PMMA \\
\hline 盓度 & & & & \\
\hline & & 0.30 & 0.50 & 0.52 \\
& & 1.00 & 1.25 & 1.40 \\
310 & 2.67 & 2.00 & 2.05 & 2.30 \\
330 & 2.89 & 2.50 & 2.75 & 3.71 \\
340 & 4.33 & 3.66 & 3.25 & 4.83 \\
\hline
\end{tabular}

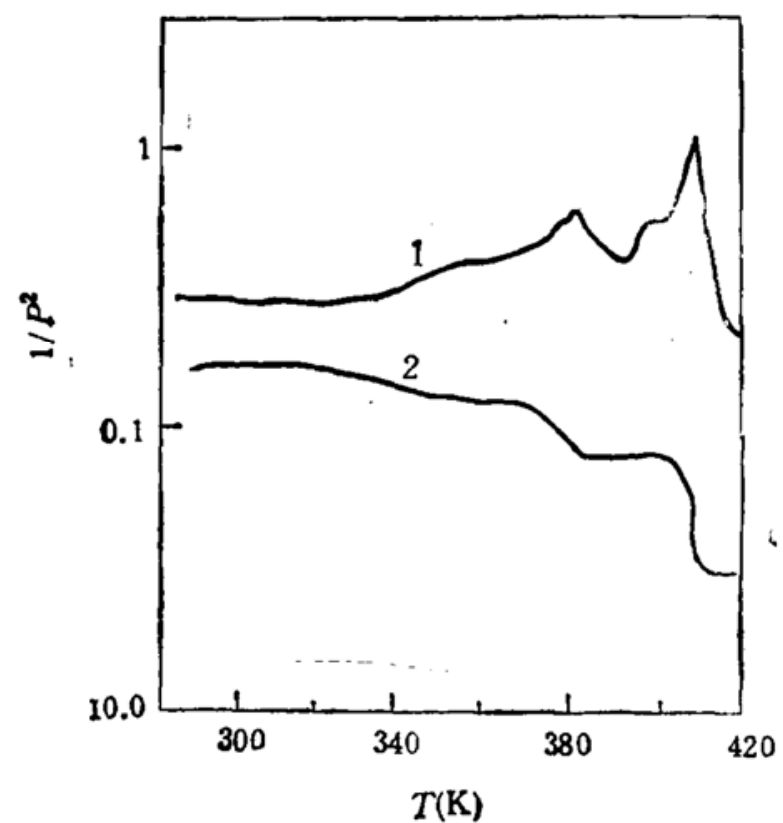

图 $3 \mathrm{PC} / \mathrm{PMMA}(90 / 10)$ 动态力学谱 1 为内耗曲线, 2 为复合模散

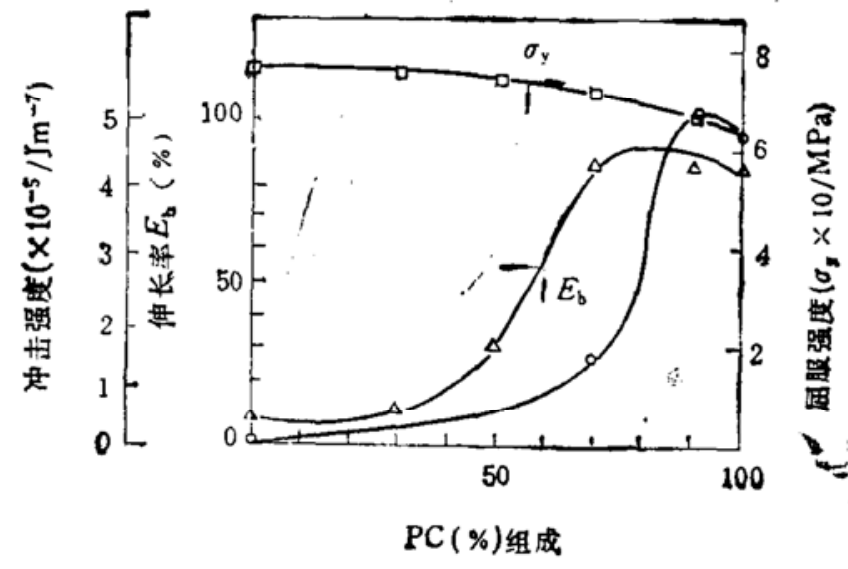

图 4 共混物力学性能与 PC 百分组成关系

\subsection{PC/PMMA 共混物动态力学徣分析}

图 3 是 PC/PMMA (组分 90/10) 样品的动态力学谱图, 从图中看到 PC, PMMA 的玻 璃化转变温度有不同程度内移, 其中 $\mathrm{PC}$ 玻璃化转变温度内移近 $14^{\circ} \mathrm{C}$, 表明 $\mathrm{PC} / \mathrm{PMMA}$ 共 混物是部分相容体系, 同时从图中还看到在 PC 转变峰邻近还出现一个小峰, 这可以解释为 PC/PMMA 共混物的界面相峰. 表明 PC 和 PMMA 分子链段在界面相有较强的作用力.

\subsection{PC/PMMA 共混物的拉伸性能分析}

在室温下, 对 PC/PMMA 共混物不同组分进行拉伸、冲击试验, 并将其抗张强度、断裂伸 
长率、冲击强度曲线变化绘于图 4 中.

从图中可以看到, 在组分 70/30-90/10 之间共混物的屈服强度、断裂伸长率、冲击强度都 在两纯组分的加合值之上, 其中断裂伸长率和冲击强度分别在 PC 的百分组成 $90 \%$ 和 $70 \%$ 处 出现极大值，而羞服强度值一直在加合值之上.

这表明,由于 PC 和 PMMA 分子链段间有较强作用力, 使共混物成为部分相容的增㓞体 系. 力学性能和动态力学分析的结果一样都支持了 ESR 谱分析得出的关于 PC 和 PMMA 分子链段间有较强作用力的结论.

\section{参来文献}

[1] Koo, K.K., lnove. T., Miyasaka. K., Polym. Eng. Sci., 1985, 25: 741-745.

[2] Kulauchi, T., Obta, T., J. Moter. Sci., 1984, 19: 1699-1703.

[3]张建中等,自旋标记 ESR 波谱的基本理论和应用,科学出版社, 北京, 1987, 40-43。 